\title{
Concept Bases: A Support to Information Systems Integration
}

\author{
Michel Bonjour, Gilles Falquet \\ University of Geneva, Centre Universitaire d'Informatique \\ 24, Rue Général Dufour \\ CH-1211 Geneva, Switzerland \\ E-mail: \{bonjour, falquet\}@ cui.unige.ch
}

\begin{abstract}
In this paper, we describe a technique for integrating heterogeneous information systems using concept bases. A concept base is build on top of each system to integrate, in order to semantically enrich its description. Each concept is described at three different levels: terminological definition, formal characterization, and representation in the underlying system. The different concept bases are then used to compare the concepts represented in the different systems and to establish correspondences between them in order to facilitate communication, cooperation, or integration. Our approach relies on results of the ConcepTerm project, dedicated to the study of concept dictionary building methodology and process. We also show that the building of a concept base can benefit database design and high-level interface definitions.
\end{abstract}

\section{Introduction}

It is currently recognized as very important to be able to integrate different information resources (among others, databases) within an organization. In fact, information is required in various applications which are becoming more and more complex and need to share information, procedures and rules. This information becomes a strategic resource of the organization that has to be used in an optimal way. However, the heterogeneity of existing systems in terms of data and process models makes very hard any attempt to share information or services.

The integration of different types of information systems (in a broad sense, ranging from specialized applications, real time data feeds, spreadsheets to classical database centred systems, knowledge bases, etc.) is the actual focus of many research projects, with a view to develop "intelligent and cooperative information systems" (the IJICIS Journal[12] is dedicated to them). These systems combine the possibilities of databases, artificial intelligence and programming languages. The notions of "distributed object system"[18] and of "knowledge communication"[25] are also suggested by the "object" research community.

In the database field, numerous research works have been undertaken to facilitate the sharing of information and services in an organization. They lead to the concept of database "interoperability" or "integration". Different projects about (mainly view or schema) integration gave rise to new approaches, described by Batini in [2] and more recently by Kambayashi in [13]. Several architectures have been defined to support a set of interoperable and/or integrated databases. Among them one can mention: distributed databases [19], multidatabases [17], and federated databases [22]. 
We have chosen the object oriented database schema integration framework to describe our approach. Concept bases are built on top of a set of schemes to integrate; they contain an enhanced semantic description of each schema and will help to establish and control equivalencies between schema elements. The first steps of the integration process are presented and a solution using a three level concept base architecture is described.

The proposed approach is based on an enrichment process using different types of complementing reference sources. Both domain and terminological knowledge are collected, resulting in a concept base able to provide a sharable knowledge space to be used in any activity involving:

- reusability, genericity, evolution of information system components

- domain learning and documentation

- semantic understanding and compliance.

The paper is organised as follows. After an introduction to the schema integration framework, chosen as an example of concept base use (section 2), we define terminology as both a theoretical and practical subject in section 3. Our approach is described in section 4, where the concept base building process is detailed. An architecture of concept base is proposed in section 5. The problem of concept translation is mentioned in section 6 . Section 7 presents methodology aspects with the ConcepTerm project, dedicated to the building process of "multilingual concept dictionaries". Finally, section 8 presents some other interesting applications of concept bases related to information systems, future research directions, and a conclusion.

\section{Schema Integration}

Most of the work done on schema integration deal only with schemes which are descriptions of data stored in a database or a file, or used by an application. Existing schema integration approaches usually decompose the integration process in several steps, the main ones being:

- Preintegration: translation of source schemes in a common data model

- Comparison: finding inter-schema correspondences

- Integration: creation of a new (global) schema using source schemes, interschema correspondences and integration rules

- Schema Transformation: modification of source schemes.

Our approach is best suited for both preintegration and (partially) comparison steps. Following Spaccapietra in [24], we'll gather these two steps together under the term "investigation". The next sections (2.1 to 2.4) describe these main steps.

\subsection{Preintegration}

The goal of this step is to translate the different schemes to be integrated into a common data model sometimes called "canonical" model [21], to extract as much information as possible concerning the schemes elements (attributes, classes, methods, etc.), and the relationships and rules existing between these elements. Generally, the data model chosen for this step is a semantic one (entity-relationship or object-oriented [15]). This preintegration step is justified, among others, by the relative semantics weakness of schemes and the heterogeneity of used data models. 


\section{Semantics of Schemes}

The emergence of modern data models and their relative ease of use ("bubbles and arrows") have promoted them as the main way to model real-world situations. However, the oversimplifying effect of any modelling effort led to a loss of semantics: a large part of the domain knowledge the designer acquired during the design phase is lost because it cannot be represented in the schema [9].

Thus, a schema, when viewed as a vocabulary or a glossary, reflects only a small part of the semantic richness of the domain it is supposed to represent. Previous works on preintegration have proposed the following formalisms to enhance schema semantics:

- Word dictionaries: linguistic information (synonyms, homonyms) are added to all of the terms (labels) present in a schema

- Name mappings: the database administrator establishes mappings from existing labels (attribute and class names) to more meaningful ones in order to improve their expressiveness.

\section{Role of the Preintegration step}

Most of the integration approaches cover this step in a partial way, only quoting it as a "precondition" to the integration step itself [24] or defining it as a "manual process" [26]. In any case the preintegration step is considered the responsibility of the database administrator (DBA).

On the contrary, we think that this step should play an important role in the integration process. For instance, knowledge stored in a schema is strictly limited to the knowledge needed by the functionality the database is developed for; other concepts of the domain without a direct (and specified) utility are ignored. Thus, the preintegration step can represent an opportunity to show the actual limits of a system with respect to an application field, by enumerating the domain concepts which aren't taken into account in this system. Consequently, it should be conducted using both domain knowledge and terminological skills. Indeed, the quality of the information added to a schema will directly determine its "semantic influence" in relation to other schemes during the comparison step. (Eick, in [6], describes the "quality" of a schema depending on the classification of objects and on its power to express rules coming from the domain).

\subsection{Comparison}

The comparison step is dedicated to finding out correspondences between schema elements by using probability or pattern matching techniques. This task is generally computer-assisted (the DBA has to validate/invalidate the correspondences suggested by the system).

Comparison is done at two levels:

1. Comparison of the Schema Elements (attribute and class names, method signatures): A probability of correspondence is computed using similarity of element names and types. A threshold is then chosen to submit only the more probable correspondences to the database administrator [11].

2. Comparison of Population: The comparison of instances allows the discovery of logical relationships (inclusion, intersection) between data and values in the databases, indicating a generalization/specialization kind of relationship between the corresponding classes and attributes. 
Some of the comparison approaches are:

- Computation of a similarity function between pairs of attributes [26]

- Computation of a semantic pertinence between elements using fuzzy logic [10].

Once established, these correspondences sometimes reveal different representations of the same concept, depending on the schema. These differences, called "inter-schema conflicts" or "semantic heterogeneity" (see[22] for a classification) are responsible for several types of problems during the integration step. Thus, the goal of the comparison step is twofold: first, to discover the inter-schema correspondences and second, to identify the conflicts and distinguish their type in order to prepare the integration step.

\subsection{Integration}

The integration process consists, generally in a semiautomatic way, to build an integrated schema starting with:

- source (original) schemes (eventually translated in a canonical data model)

- inter-schema correspondences (coming from the comparison step)

- integration rules (depending on the method and data models used).

\subsection{Schema Transformation}

This step is mainly present in case of view integration. The recent approaches (federated ones) don't allow later modifications of source schemes, to preserve the local environment (data, applications) of the databases composing the federation and to guarantee their autonomy.

\section{Terminology and Concept Bases}

\subsection{Foundation of Terminology}

Among the numerous meanings of the word terminology, we note the following ones (coming from [5]) which justify the usage of terminology in our work:

1. Description of an Existing Terminology Content. This is a systematic study of an existing terminological reality. It consists in making an inventory of the terms of a domain and to organize them as a "domain tree". Each term is defined, described, its relationships with other terms are noted. This is an analytic activity describing the lexical and conceptual aspects of a domain in order to establish a dictionary.

2. Set of Terms Related to a Domain. This is a coherent set of terms reflecting the conceptual system of a domain. It constitutes the vocabulary used by experts of the domain. In that sense, it represents a particular language.

Thus, the approach we propose supports the schemes preintegration task by a terminological activity (in the sense of 1) to establish one or many terminologies (in the sense of 2) of a domain. These terminologies will then constitute the core of concept bases.

Terms, Concepts, and the Domain

A term is the significant unit which uniquely defines a concept within a domain. The term is made up of one word (simple term) or several words (complex term).

A concept is a representation of a reality's aspect isolated by the mind. This is a unit of thinking based on a set of properties attributed to an object or a class of objects. 
The domain may be an activity, a problem, a discipline in which a language called "particular language" (as opposed to the general language) exists and is used by experts of the domain.

The relationships between term, concept and domain are shown in Fig. 1:

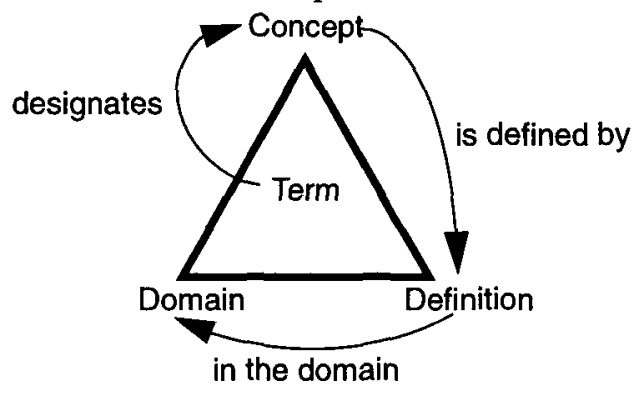

Fig. 1 Term, Concept and Domain relationships

Theoretical Aspects of Terminology

Terminology studies the designation process, the organization of concepts in classes and systems, the conceptual structuration of a domain. It also studies lexicons and nomenclatures, their structure and their use. Moreover, terminology uses results from other fields such as taxonomy, logic, linguistic, etc.

The role of the definition is very important in terminology. A concept definition must permit to distinguish it from any other. For that purpose, the existing relationships between a concept and the rest of the domain must be enumerated and its defining characteristics values specified.

\subsection{Terminology Building Method}

The "classical" approach of a terminologist building the terminology of a domain begins with a precise delimitation of this domain. Complementing sources of information are then collected. The goal is to collect documents acting as domain reference sources like dictionaries, regulations or any other accepted and up-to-date documents.

The domain tree materializes the generic-specific relationships existing between the concepts. It is augmented each time a new concept is found and/or when analysing its definition. An other use of the domain tree is to delimit a domain by enumerating the set of its concepts.

The main data representation tool of the terminologist is the term record. A term record represents a concept, using a set of textual fields filled with the concept corresponding values. Apart from the concept's definition, terminological, linguistic and other information concerning the making of a record are also noted (source mention, reliability degree, etc.). A set of such records can be stored in a database to form a terminological database (or term bank).

\subsection{From Terminological Bases to Concept Bases}

A concept base is a database dedicated to the storage and management of a set of concepts related to a common domain. The information stored for each concept is composed of its definition and its relationships with other concepts (in particular the generic/specific relationship). 
Since the definition of a concept stored in a terminological database is expressed in a natural language form, it is not easily machine processable. If one wants to automatically manage concepts, it is necessary to express their definition in a formal way. This is usually accomplished by choosing a set of characteristics, the values of which are necessary and sufficient to distinguish each concept from the others. The formalized definition of a concept is then expressed by specifying the values it takes for each characteristics.

For example, during our study of the furniture domain (see section 7), the following defining characteristics have been collected: accessories, parts, dimension, shape, perception, place, localization, material, main usage, secondary usage. Thus, when establishing the furniture terminology, each concept has been defined and described by giving the value it takes for these common properties.

The formalization of concepts' definitions makes it possible to implement functions such as finding a concept on the basis of characteristics or comparing concepts and express what makes them different.

\section{A Terminological Approach to Schema Preintegration}

We focus our attention on the first step of the integration process, called "preintegration". As explained in section 2.2, this step is dedicated to the analysis of the source schemes in order to extract their semantic content. To this end we propose to create a concept base on top of each schema to integrate. Such a concept base aims at (re-)building the conceptual dictionary of the domain currently modelled by a schema.

The goal is to build a concept description as complete as possible to better inform the "integrator" on the meaning of concepts existing in the several databases to integrate. The more precise the concepts descriptions are, the more reliable will be their comparison, thus avoiding to consider as equivalent concepts which are only superficially (at the schema level) equivalent.The main advantage of a concept base over a simple terminological database is its formal representation of concepts that will ease automatic processing during the schema comparison step.

\subsection{Terminology Building}

To build a concept base it is necessary to first establish the terminology of the schema's domain.The first task is to identify the domain and to establish a list of all its terms. The schema may be a good starting point, its study showing the partial organization of the concepts stored in the database. Nevertheless, the schema only covers a subset of the domain (see point 2.1); it must be considered as an incomplete source, so other sources (like dictionaries or regulations) are necessary to reveal missing concepts. These sources provide precise (and accepted) definitions of the different concepts as well as the statements of rules governing relationships between concepts. Their textual orientation allows also to gather other information like synonyms, attribute domain, context, etc.

When building the domain tree (conceptual structure), concepts that were not present in the schema may appear. For example, the concept of "teacher" is not taken into account in a database containing classes Professor and Lecturer, both subclasses of Employee. However, the concept of teacher owns characteristics which are different from other employees like technicians or secretaries. 


\subsection{Concept Base Building}

The main task involved in concept base building is to find properties that characterize the domain's concepts. These properties are sometimes ignored at the data schema level, because they seem to be implicit in the framework of a database. For example, the usage is a common property of any piece of furniture; nevertheless, it is unlikely that it will be explicitly given for each entity in a furniture database composed of classes like "seat" and "wardrobe". Indeed, each of these classes represents a set of furniture sharing an implicit value of usage ("to seat", "to arrange"). This is why the discovery of characteristics must use the definitions stored in the terminological database. Although this task cannot be fully automated, an array of computerized tools for browsing terms definitions, doing textual searches, etc. greatly enhance human capabilities.

\section{Architecture and Functions of the Concept Base}

The concept base we propose to build on top of each schema must not only store concepts characteristics, it must also support activities like: terminology building, formal characteristics finding, concepts comparison, and finally schema integration. For these purposes each concept is described at three levels, namely: terminological, formal characteristics, and representation in the underlying schema.

\subsection{Terminological Information and Functions}

The terminological description of a concept includes common terminological information such as: term: definition, position in the concept hierarchy, related and dependent concepts, linguistic context (the set of verbs, actions, adjectives, etc. which may be associated to the term in the domain language).

As mentioned earlier, finding out an appropriate set of characteristics for the concepts of a domain is an exploratory process that requires browsing, navigation, and search tools as well as more sophisticated tools to establish statistics and concordance. This is why we chose to represent terminological information in a hypertext structure. Each hypertext node stores textual information about a concept (term, definition, etc.), while hyperlinks represent semantic relationships between concepts (generic/specific, existential dependency, "part-of", etc.).

The navigation capabilities of hypertext systems, combined with global associative search and easy link building make it an efficient support to knowledge acquisition and exploration during the base's building phase, and a helpful tool for domain understanding and learning during both integration and exploitation.

In the ConcepTerm prototype this hypertext layer is implemented as a set of HyperCard $^{\circledR}$ "stacks", with specialized exploration tools programmed in the HyperTalk ${ }^{\circledR}$ scripting language.

\subsection{Formal Characteristics}

In order to be automatically processed, the description of each concept must be based on a common "mould", its elements having to be as elementary as possible.

The formal characterisation of a set of concepts is based on the finding of common properties systematically present in each concept description, but with different values. Since characteristics values can themselves have a complex structure we chose to represent the formal definition of concepts as complex objects in an object-oriented data model. For instance the concept of "bergere" (in the furniture domain) can be represented by the following complex object: 


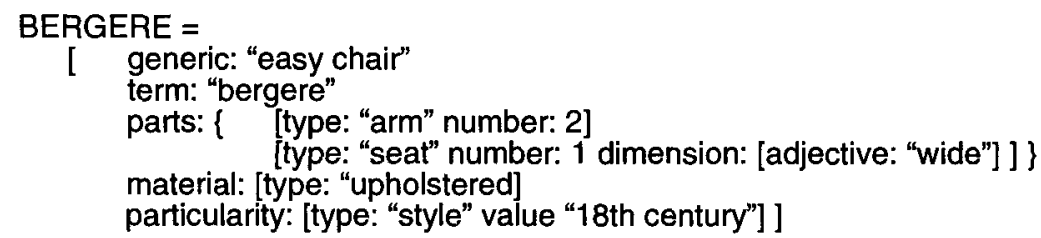

In addition, an object-oriented data model with complex values is well adapted to the specification of operations and methods acting on a concept base. Some of these operations are:

- complex properties inheritance, which needs more complicated process than the usual duplicating of generic inherited values

- comparison of concepts based on properties values

- search of equivalent concepts in another concept base.

The formal characteristics layer of the ConcepTerm prototype is based on the F2 object-oriented database management system [7] and its F2Concept extension [8][9]. The whole system is implemented as a client/server architecture, the hypertext layer acting as a graphical user interface and the F2 DBMS as a concept management system.

\subsection{Mapping to Underlying Schemes}

Generally, databases aim at storing a (computer) representation of a subset of the complete extension of a concept. For example, in a College database, we'll find the subset of the "student" concept extension corresponding to the students registered in that College. Each individual student will be represented by a tuple of values expressing his $(\mathrm{h}$ er) name, first name, degree, etc. On the other hand, some concepts will not be represented by data but by a process, an integrity constraint, a deduction rule (if available) or even by a combination of data/process/constraint/rules.

The description of a concept's representation in a database is given by a function which associates to each concept:

- its representation in terms of object-oriented schema elements, describing the form taken by an instance of the concept within the underlying database

- several instance management procedures describing the primitives operations to create or delete an instance, to search (access to) any or all instances, etc. These procedures are only specified if they are not implied by the representation schema.

The following list enumerates a set of elementary representation schemes we have identified:

a) Concept $\rightarrow$ (Virtual) Class

In this simplest case, a concept is mapped to a class; an object represents an instance of the concept. The operations defined on instances correspond to those on objects (create, update, delete).

If the class is virtual, i.e. computed using one or many other classes (like a relational view), the operations of instance creation and deletion may be more complex and have to be explicitly described.

Example: The concept of "literary works" may be represented by a virtual class derived from the Author and Book classes: 


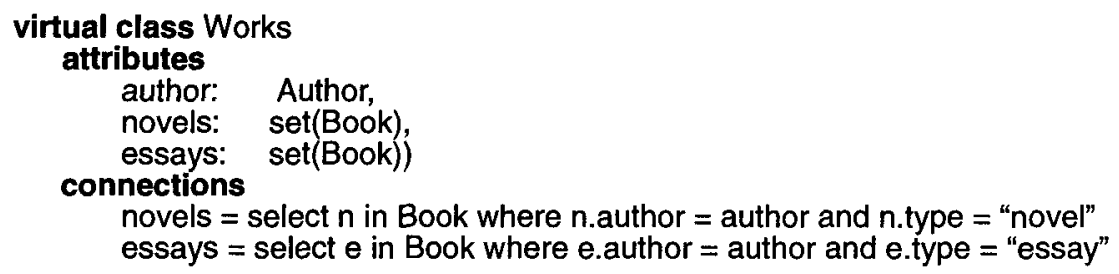

To find the works instance corresponding to the author "Hugo", we need to select an Author object and to join it with objects from the Book class. The deletion of this instance would require the deletion of all books of this author.

b) Concept $\rightarrow$ Attribute

Each instance of the concept is represented by a value of the same attribute. It should be noted that the existence of an instance depends on the existence of an object in the class owning the attribute.

Example: The concept of "mother tongue" may be represented using an attribute mother_tongue in the Person class, its set of values defined in class Language. To create an instance of mother tongue amounts to give a value of mother_tongue for an existing entity of the Person class.

When an attribute is calculated (not stored), the creation and suppression operations have to be explicitly defined. Note that concepts represented by attributes are always dependent on other concepts and act as links between concepts.

c) Instance $\rightarrow$ Class or Set

An instance of a concept may appear as a set of objects of the same type. In that case, the instance will be represented by a "container" which should be a class extension or a selection of objects.

Example: The market prices of stock $S$ (the values of stock $S$ during a period) may be represented as

class $S$

attributes date, value, exchanged_volume

or as

select $x . d a t e, x . v a l u e, x . e x c h a n g e d \_v o l u m e$ from $x$ in Exchange_daily_record where label = "S".

d) Concept $\rightarrow$ Integrity Constraint Schema

Some concepts do not generate any data, i.e. their instances are not explicitly stored in the database, but they act upon its content either as constraints or as data transformations rules.

Example: The concept of "land occupation" may be represented by a generic constraint:

for all $p$ in PieceOfLand p.surface_built / p.surface $<t$

An instance of this concept corresponds an instantiation of the constraint formula, i.e. a formula obtained by replacing all free variables by constants, as in

for all $p$ in PieceOfLand: p.surface_built / p.surface $<0.25$

e) Concept $\rightarrow$ Process Schema

When a concept is an action, its representation may be a process (method, transaction, or application). An instance of such a concept corresponds to the execution of this process. 
Example: The concept of promotion is represented by a method:

promote (toGrade, date)

of class Student. The creation of an instance of promotion corresponds to the execution of the method on an object with actual parameters, like in:

joe.promote(G6, 1994-06-31)

The ability to delete an instance depends on the existence of an inverse process; in the same way, the access to existing instances is realized by querying the process execution history (to be recorded in a journal). The architecture of a concept base is illustrated in Fig. 2 below.

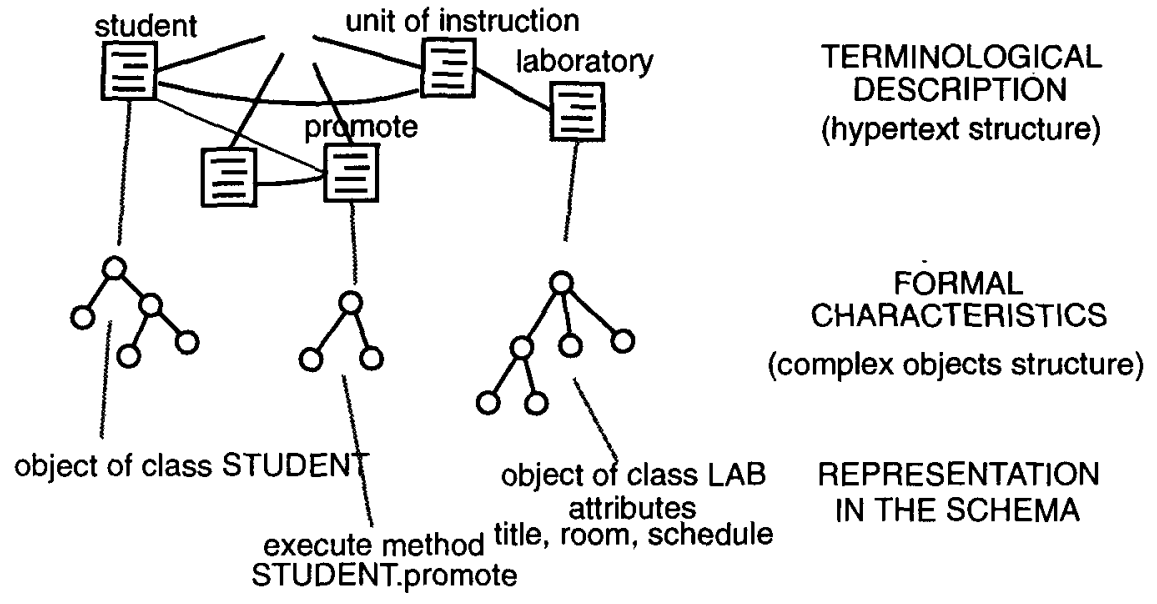

Fig. 2 A three level Architecture of a Concept Base

\section{Concepts Comparison and Translation}

The building of a concept base on top of each schema to integrate allows a two-levels comparison of these schemes: First, on the represented concepts and second, on the schema elements and population. Concepts comparison is necessary since the same term may designate different concepts, depending on the system. For instance, although there exists a class Student in two schemes to compare, the concept of student may be different in the two systems; it is thus necessary to exactly understand the similarities and differences between these concepts before trying to integrate or translate underlying schema elements.

The comparison of concepts is based on their characteristic values and on the existence of well established equivalencies between these values. An equivalence function is defined for each formal characteristic. Thus, an equivalence function may be "simple", consisting of value comparison according to their identity or to a value scale. On the other hand, some functions may consist of complex comparison and calculation to determine equivalence.Examples of equivalence functions:

- "Simple" function: equivalence between dimensions (tall, small): This function is based on an existing order between dimension values (ex: small $\leq$ tall)

- "Complex" function: Equivalence between the usage of pieces of furniture (to store books, to store clothes, to seat, to sleep). In that case, the function has to 
be defined on both the action (to store, to seat, to sleep) and the objects on which the action is acting (books, clothes)

- Function with translation: equivalence between characteristics values express in different language or with synonyms, e.g., usage.action: to sleep (English) is equivalent to usage.action: schlafen (German). In that case a set of simple term equivalencies (like to sleep $\approx$ schlafen) between the two languages must be provided.

Once equivalencies between concept of different schemes have been discovered, inter-schema correspondences can be established using the schema representation of these concepts and validated at the population level. Generally, equivalencies will not be perfect, indicating that some extra work has to be done to find correct inter-schema correspondences. In this respect one can mention concepts which are not completely equivalent although they have the same representations in the underlying schemes. This is the kind of semantic conflict that cannot be detected at the schema level.

\section{Methodology Aspects: The ConcepTerm Project $^{1}$}

ConcepTerm is a research project dedicated to the creation of encyclopedic and multilingual dictionaries of concepts. The building and management process of such dictionaries is studied. ConcepTerm is a collaboration between database specialists and terminologists from the University of Geneva. The interdisciplinary nature of the project aims at putting together database and terminological formalisms, in order to define a set of generic methods and tools to support the building of a new generation of computerized dictionaries.

The architecture defined for this project is based on concept bases describing the conceptual spaces of a particular domain in four different languages. In this case, the four languages should be seen themselves as four different domains, each one with its own domain tree and concept space. These concept bases are used to physically store the terminologies of the domain; they were realized with terminological techniques assisted by computer tools.

Building of the Dictionary " 0 "

The domain of furniture was chosen to serve as conceptual space for our first dictionary. This domain was chosen for different reasons, including the fact that everybody knows it and that documents describing the domain are abundant.

A first set of defining characteristics was drawn up by analysing the definitions found in general dictionaries and reference books of the domain. Concepts definitions, together with their characteristics were entered in a hypertext system. Using exploration and analysis tools we were able to further refine the structure of characteristic values until they were broken into sufficiently simple pieces (words or numbers). Once this structure was well established concepts descriptions were entered into an F2 database linked to the hypertext system. This resulted in the building of four separate dictionaries of furniture (in French, English, German and Italian) of approximately 250 concepts each.

The next step was to establish equivalencies between defining characteristics values for each pair of languages. Once again, an exploratory approach was taken to create equivalence relations between simple values stored in the dictionaries.

1. Supported by Swiss FNRS. See [4] for a description of the project 
Finally an interactive tool to find concept equivalencies has been designed, that is based on a set of value equivalence relations. We are currently studying the definition and implementation of more complex equivalence functions.

\section{Development of Computer Tools}

The dictionary " 0 " has been used as a test platform for the data structures, tools and interfaces developed for ConcepTerm. It allowed us to recognize the different functionalities needed by the users during both the dictionary building process (expert users) and query process (non-expert users). These needs have also been considered for both mono- and multilingual usage.

\section{Conclusion and Future Directions}

We have presented a possible solution to the investigation step of the database schema integration process. This solution proposes to use terminological techniques during the preintegration step. Each schema is semantically enriched by building a concept base on top of it. These concept bases will then support the comparison of concepts and the establishment of correspondences between schema elements. The main advantages of this approach are:

- Better inter-schema correspondences based on domain knowledge instead of schema information

- Clear distinction between concepts comparison and schema comparison

- The discovery of possible semantic conflicts that do not appear at schema level (when different concepts have the same representation)

- The building of a very high level, yet computer based, description of an information system.

Moreover, other activities where a complete and precise description of a domain is necessary may gain advantages from such a concept base:

Design Dictionaries: A concept base may be used to build and manage a design dictionary, frequently called "encyclopedia" or "repository". The CASE tools success has shown the interest for that kind of dictionaries. Unfortunately, it has also shown that a simple "schema collection" support system is not sufficient to help a team of designers work together on a project. A concept base should allow the sharing of a terminology, based on precise definitions coming from accepted sources and improved with more personal information like abbreviations or synonyms used by the different designers during their activities.

High-level Interfaces: The recent World Wide Web project [16] and its growing success show that both the electronic (wide) publication of information and the access to information are becoming major concern of organizations. Consequently, databases, as potential sources of huge information volumes, have to participate to this process. A concept base seems to be a possible support to the publication of a database, by presenting to the "external" world a semantically improved schema (multi-language descriptions, definitions, synonyms). Mappings defined in the concept base should be used to access the underlying system.

The remote users have to be considered as a new type of users for a database. They ignore some aspects implicitly known by the "local" users, members of the organization. Thus, a concept base representing a complete terminology of the domain and clear- 
ly describing these implicit aspects should improve the navigation and the information search process in conventional databases.

\section{Future Research Directions}

Our future research directions include the definition and implementation of more complex equivalence functions in a semiautomatic comparison system. We have also observed that it would be valuable to include encyclopedic information (like examples, properties, pictures, etc.) within the description of concepts in order to help their understanding and comparison, in particular for abstract and complex domains. Finally, we continue to study hypertext and hypermedia interface for concept bases and to develop the tools needed to build and manage such systems.

\section{Acknowledgments}

We are deeply grateful to Prof. Michel Léonard for giving us the opportunity to work on this subject and for fruitful discussions. We also thank Bruno de Bessé for exposing us to terminology and Atika Laribi and Ian Prince for their precious comments.

\section{References}

1. T. Barsalou, G. Wiederhold, "Knowledge-directed Mediation between Application Objects and Base Data", In Data and Knowledge Base Integration (ed. S M Deen).

2. C. Batini, M. Lenzerini, S.B. Navathe, "A comparative analysis of methodologies for database schema integration”, ACM Computing Surveys, 15, 1986.

3. M.W. Bright, A.R. Hurson, "Linguistic support for semantic identification and interpretation in multidatabases", In Proceedings of First International Workshop on Interoperability in Multidatabase Systems, Kyoto, 1991.

4. ConcepTerm: "Construction de dictionnaires encyclopédiques de concepts multilingues et informatisés", projet FNRS no. 12-28834.90. Common project of CUI (database) and ETI (terminology).

5. B. de Bessé, "Stage de terminologie", Ecole de Traduction et d'Interprétation, Genève, mars 1991.

6. C. F. Eick, "A Methodology for the Design and Transformation of Conceptual schemes", In Proceedings of 17th VLDB, Barcelona, 1991.

7. Th. Estier, G. Falquet."F2: an Evolution Oriented Database System, CUI Tech. report no. 69, Centre Universitaire d'Informatique, Genève, 1993.

8. G. Falquet, J. Sindayamaze, M. Bonjour, M. Léonard. "F2Concept, un Modèle intégrant la Description de la Compréhension et l'Extension des Classes d'Objets", Proc. VIIèmes Journées Bases de Données Avancées BD3, Lyon, 1991.

9. G. Falquet, M. Léonard, J. Sindayamaze. "F2Concept: a Database System for Managing Classes'Extensions and Intensions.", In Proc. of the 3rd EuropeanJapanese seminar on Information Modelling and Knowledge Bases, Budapest, 1993.

10. P. Fankhauser, M. Kracher, E.J. Neuhold, "Semantic VS. Structural Resemblance of Classes", SIGMOD RECORD, Vol 20, No 4, Dec. 1991.

11. S. Hayne, S. Ram, "Multi-User View Integration System (MUVIS): An Expert System for View Integration", In Proceedings of 6th Conference on Data Engineering, 1991. 
12. International Journal of Intelligent \& Cooperative Information Systems, ed. M.P. Papazoglou, T.K. Sellis.

13. Y. Kambayashi, M. Rusinkiewicz, A.P. Sheth, "First International Workshop on Interoperability in Multidatabase Systems", Kyoto, 1991.

14. M. Kaul, K. Drosten, E.J. Neuhold, "ViewSystem: Integrating Heterogeneous Information Bases by Object-Oriented Views", In Proceedings of DATA Engineering, Los Alamitos [etc.]: IEEE, cop. 1992.

15. W. Kim, N. Ballou, J.F Garza, D. Woelk, "A Distributed Object Oriented Database System supporting Shared and Private Databases", ACM Trans. on Information Systems, Vol 9, No 1, 1991.

16. E. Krol, M. Loukides (eds). The Whole Internet: user's guide and catalog, Sebastopol: O'Reilly, 1992.

17. W. Litwin, L. Mark, N. Roussopoulos, "Interoperability of Multiple Autonomous Databases", ACM computing surveys, Vol 22, No 3, Sep.1990.

18. F. Manola, S. Heiler, D. Georgakopoulos, "Distributed Object Management", International Journal of Intelligent \& Cooperative Information Systems, Vol 1, No 1, March 1992.

19. T. Ozsu, P. Valduriez, "Principles of Distributed Database Systems", Prentice Hall ed., 1989.

20. M.P. Papazoglou, S.C. Laufmann, T.K. Sellis, "An Organizational Framework for Cooperative Intelligent Information Systems", International Journal of Intelligent \& Cooperative Information Systems, Vol 1, No 1, March 1992.

21. F. Saltor, M. Castallanos, M. Garcia-Solaco, "Suitability of data models as canonical models for federeted databases", SIGMOD RECORD, Vol 20, No 4, Dec. 1991.

22. A.P. Sheth, J. Larson, "Federated Database Systems for managing heterogeneous, distributed and autonomous Databases", ACM Computing Surveys, Vol 22, No 3, 1990.

23. A.P. Sheth, V. Kashyap, "So Far (Schematically) yet So Near (Semantically)", in Proceedings of Interoperable Database Systems, Lorne, Australia, 1992.

24. S. Spaccapietra, C. Parent, Y. Dupont, "Model Independant Assertions for Integration of Heterogeneous schemes", VLDB Journal, 1, ed. Dennis McLeod, 1992.

25. C.C. Woo, F. Lochovsky, "Knowledge Communication in Intelligent Information Systems", International Journal of Intelligent \& cooperative Information Systems, Vol 1, No 1, March 1992.

26. C. Yu, W. Sun, S. Dao, "Determining Relationships among Names in Heterogeneous Databases", SIGMOD RECORD, Vol 20, No 4, Dec. 1991. 